\title{
ECOLOGIA TRÓFICA DE ONÇA-PARDA (Puma concolor) EM PAISAGEM AGRÍCOLA
}

\author{
Carla Gheler-Costa* \\ Gabriel Pavan Botero** \\ Lais Reia*** \\ Leticia de Cassia Gilli**** \\ Fabio Henrique Comin ***** \\ Luciano Martins Verdade ${ }^{* * * * * *}$
}

RESUMO: Os carnívoros são importantes componentes ecológicos dos ecossistemas, pois controlam a abundância, a distribuição e a diversidade das populações de suas presas. Desta forma, são considerados como espécies-chaves na manutenção e restauração da diversidade e da resiliência dos ecossistemas. Nesse grupo, a onça-parda (Puma concolor) é a espécie de mamífero silvestre mais amplamente distribuída do hemisfério ocidental, porém, suas exigências em tamanho de área de vida e alimentação mostram-se um fator de restrição para sobrevivência da espécie por fatores como pressão de caça, diminuição de presas e desmatamento. Este estudo teve como objetivo avaliar a dieta do $P$. concolor em uma paisagem silvicultural no Estado de São Paulo. A paisagem é formada pela presença de remanescentes de cerrado, plantações de eucalipto e áreas de pastagens. Para análise da dieta foram coletadas, triadas e analisadas 103 amostras de fezes de $P$. concolor. Os resultados indicam grande consumo de aves e mamíferos de pequeno porte, diferindo da alimentação da espécie em ambientes naturais onde os mamíferos são o principais itens alimentares. Esta variação na dieta confirma a onça-parda como predador generalista, que consume as presas disponíveis sem apresentar preferência por determinadas espécies.

PALAVRAS-CHAVE: Dieta; Carnívoros; Agroecossistema; Conservação.

\footnotetext{
Doutora em Ecologia Aplicada (USP), Docente do Programa de Pós-graduação em Ciências e Tecnologia Ambiental, Universidade do Sagrado Coração (USC), Bauru (SP), Brasil. E-mail: cgheler@gmail.com

** Biólogo (USC), mestrando na Universidade Regional do Cariri, Crato (CE), Brasil.

${ }^{* * * *}$ Mestre em Zoologia (UNESP), Botucatu (SP), Brasil.

${ }^{* * * *}$ Mestre em Ciência e Tecnologia Ambiental, (USC), Bauru (SP), Brasil.

***** Doutor em Ciências (USP), Docente do Centro de Ciências Exatas e Sociais Aplicadas, Universidade do Sagrado Coraçãa (USC), Bauru (SP), Brasil.

${ }^{* * * * * *}$ Docente do Centro de Energia Nuclear na Agricultura (CENA/USP), Piracicaba (SP), Brasil.
} 


\title{
TROPHIC ECOLOGY OF THE PUMA (Puma concolor) WITHIN AN AGRICULTURAL ECOSYSTEM
}

\begin{abstract}
Carnivores are important ecological components of ecosystems since they control abundance, distribution and diversity of prey populations. They are keyspecies in the maintenance and restoration of diversity and resilience of ecosystems. The Puma concolor (onça-parda) is a forest mammal with wide distribution in the western hemisphere. Its requirements in area size, life span and feed are a restriction factor on the survival of the species due to hunting pressure, prey decrease and deforestation. Current analysis evaluates the diet of $P$. concolor in a silvicultural environment in the state of São Paulo, Brazil. The environment comprises savannah remnants, plantations of eucalyptus trees and pasture. One hundred and three excrement samples were retrieved, selected and analyzed. Results show the consumption of great amounts of fowl and small-size mammals. Feed differs from that of species within their natural habitat where preyed mammals are the main items. Diet variation confirms the puma as a generalist predator consuming available preys without any preference for certain species.
\end{abstract}

KEY WORDS: Diet; Carnivores; Agro-ecosystem; Conservation.

\section{INTRODUÇÃO}

Entre os grupos de vertebrados, os mamíferos carnívoros têm sido utilizados como espécies alvo em diversos projetos conservacionistas e são considerados como espécie bandeira e espécie guarda-chuva, por ocuparem o mais alto nível trófico dentro da cadeia alimentar (predadores de topo) e desempenharem importante papel na dinâmica dos ecossistemas (TERBORGH et al., 1999; WILLIAMS; MARTINEZ, 2004; SCHIPPER et al., 2008; RONDININI et al., 2011; DOBROVOLSKI et al., 2013). Mamíferos carnívoros, atualmente, apresentam populações pequenas e muitas vezes em declínio, classificados com algum grau de ameaça de extinção, consequências muitas vezes das alterações na paisagem causadas pelas atividades humanas, como a agricultura (MARTINELLI; FILOSO, 2008; VERDADE et al., 2012; 2015)

A maioria dos carnívoros possui especializações para se alimentar exclusivamente de carne, enquanto outros incluem alimentos vegetais em suas dietas, como frutos, 
sementes e gramíneas. A estrutura da comunidade de carnívoros é influenciada tanto pela abundância relativa e diversidade de presas, como pela configuração do habitat onde vivem, e também sua ação sobre as presas pode influenciar toda a dinâmica do ecossistema (RABINOWITZ; WALKER, 1991; SANDOM et al., 2013, RIPPLE et al., 2014). O estudo alimentar da maioria dos mamíferos carnívoros revela a composição de presas na natureza, assim como sua abundância relativa e, desta forma, fornece dados que propiciam o entendimento acerca da estrutura trófica de todo um ecossistema (TERBORGH et al., 1999; HEALTH et al., 2014), inclusive daqueles cuja paisagem foi alterada.

Aonça-parda (Puma concolor) éa espécie de mamífero silvestre mais amplamente distribuída no hemisfério ocidental, estendendo-se do Canadá à Argentina, sendo a segunda maior espécie de felino das Américas (EISENBERG; REDFORD, 1992). O tamanho corpóreo e o tipo de dieta exigem que a espécie ocupe grande área de vida para assegurar sua sobrevivência, variando de $69,9 \mathrm{~km}^{2}$ até $248 \mathrm{~km}^{2}$ (CRAWSHAW; QUIGLEY 2002; MAZZOLLI, 2010). Ocorre em habitats diversos como desertos, florestas tropicais e florestas de coníferas (WOLFF, 2001; VIDOLIN, 2004), mas a pressão de caça, a diminuição de suas presas e o desmatamento pode restringir sua distribuição territorial (MAZZOLLI, 2010). Atualmente, a onça-parda tem sido registrada em ambientes bastante antropizados, incluindo paisagens agrícolas e áreas suburbanas (KERTSON et al., 2011; MIOTTO et al., 2012; MAGIOLI et al., 2014). No Brasil, atualmente, a maior causa do declínio das populações de mamíferos carnívoros é a redução ou perda de habitat ocasionada pela ação antrópica por meio da expansão agrícola, pecuária, exploração mineral, e urbanização (SANTOS et al., 2004; MIOTTO et al., 2012; MAGIOLI et al., 2014).

A crescente modificação de ambientes naturais pelo homem pode interferir na estrutura das populações de carnívoros e, consequentemente, influenciar tanto na dinâmica do ecossistema, como, nos seus hábitos alimentares. Na dieta da onçaparda em ambientes naturais, os mamíferos são o grupo mais abundante, seguidos por répteis e aves. Entre os mamíferos, destacam-se os roedores e espécies como o quati (Nasua nasua, Procyonidae), o furão (Galictis cuja, Mustelidae) e o cachorrodo-mato (Cerdocyon thous, Canidae) (VIDOLIN, 2004). Segundo GonzalezBorrajo et al., (2016), a ecologia de Puma concolor tem sido bem estudada em 
áreas temperadas da América do Norte, no entanto, ainda é relativamente pouco conhecida na região neotropical (SCOGNAMILLO et al., 2003; NOVACK et al., 2003; 2005; MIOTTO et al., 2007; 2011; 2012; MAGIOLI et al., 2014). Nesse sentido, o conhecimento de processos ou interações e mecanismos ecológicos, naturais ou induzidos pelo homem são essenciais para a conservação da vida silvestre. Portanto, diante deste cenário, o presente estudo teve como objetivo principal analisar a dieta de onça-parda em uma paisagem silvicultural do Estado de São Paulo, na tentativa de estabelecer um padrão na dieta da espécie e as possíveis estratégias e preferências com relação às presas consumidas.

\section{MATERIAL E MÉTODOS}

\section{1 ÁREA DE ESTUDO}

A amostras de fezes de $P$. concolor foram coletadas em estradas e carreadores na Fazenda Três Lagoas $\left(23^{\circ} 22^{\prime} 0\right.$ " S e $48^{\circ} 28^{\prime} 0^{\prime \prime}$ O) e na Fazenda Arca $\left(23^{\circ} 20^{\prime} 0^{\prime \prime}\right.$ S e $\left.48^{\circ} 27^{\prime} 30^{\prime \prime} \mathrm{O}\right)$, situadas no município de Angatuba, na bacia hidrográfica do Alto Paranapanema, Estado de São Paulo. Nestas fazendas existe uma grade do PPBio (MARTIN et al, 2012), contendo 30 pontos distantes $1 \mathrm{Km}$, e a partir destes pontos foram utilizadas estradas e carreadores em busca de amostras fecais (Figura 1). As coletas foram realizadas durante os anos de 2013 e 2014. As duas fazendas são fronteiriças e suas áreas têm, respectivamente, 3.209,93 ha e 1.122,77 ha. Na década de 1970 , a vegetação nativa destas fazendas foi suprimida para a implantação de pastagens exóticas para criação de gado de corte. Entre agosto de 2006 e novembro de 2007, 2.223,9 ha de pastagens exóticas (Brachiaria spp.) da Fazenda Três Lagoas foram convertidas em eucaliptais. As demais áreas de pastagem foram abandonadas a fim de recompor a Reserva Legal (586,52 ha) e as Áreas de Preservação Permanente $(269,23 \mathrm{ha})$ da fazenda. O mesmo ocorreu na Fazenda Arca no período de setembro de 2007 a maio de 2008, resultando em 721,9 ha de eucaliptais, 293,98 ha de Reserva Legal e 66,6 ha de Área de Preservação Permanente (MARTIN et al., 2012). O clima da região é do tipo subtropical apresentando temperaturas médias nos meses 
mais quentes superiores a $22^{\circ} \mathrm{C}$ e nos meses mais frios de aproximadamente $17^{\circ} \mathrm{C}$ (MARTIN et al., 2012).

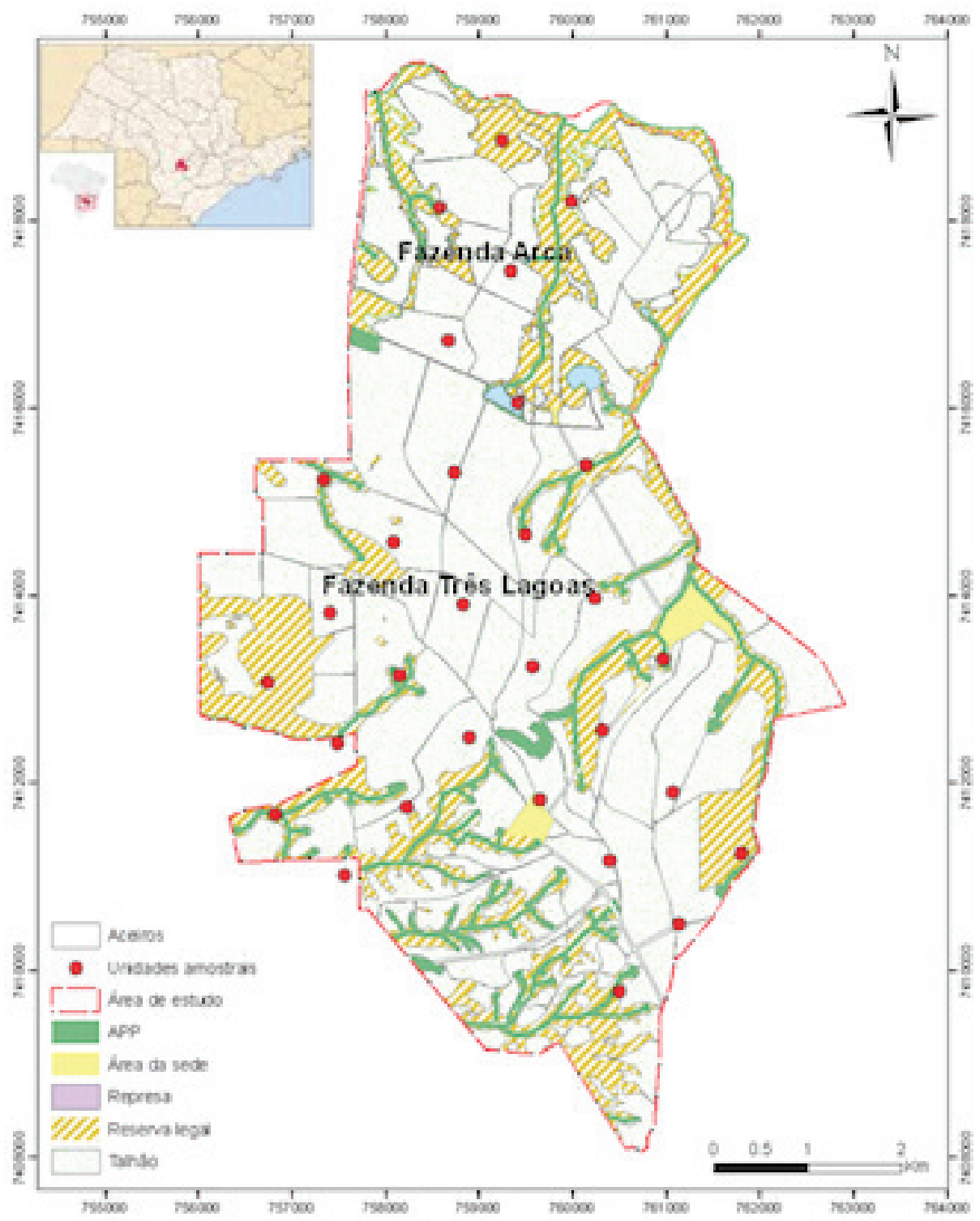

Figura 1. Fazendas Arca e Três Lagoas, localizadas no município de Angatuba, bacia do Alto Paranapanema, Estado de São Paulo. 


\subsection{COLETA DAS AMOSTRAS}

As amostras foram coletadas ao longo de 24 meses, tendo início em agosto de 2013 e término em julho de 2015, com visitas quinzenais a campo com pelo menos quatro dias de coleta para que toda as áreas de estudo pudessem ser percorridas.

Todas as amostras encontradas foram coletadas na trilha estabelecida em carreadores entre as áreas de vegetação nativa e talhões de eucalipto, eem carregadores entre talhões de eucalipto. Cada amostra foi armazenada individualmente em sacos plásticos e identificadas com uma ficha de coleta contendo dados como tipo de substrato, coordenadas geográficas, rastros indicativos, condição estrutural, cobertura e uso local da terra. Todas as amostras foram fotografadas em campo e armazenadas sob refrigeração. Toda a coleta de material foi realizada com utilização de luvas, máscaras e óculos de segurança para evitar contaminação por diferentes patógenos entre eles toxoplasmose. Para auxiliar na identificação e na tentativa de individualização dos espécimes foram coletas informações morfológicas das amostras fecais (calibre, comprimento e aspecto), além das medidas de pegadas, quando presentes.

\subsection{ANÁLISE DO MATERIAL COLETADO}

As amostras fecais foram lavadas em água corrente, utilizando duas peneiras de diferentes malhas $(1,0 \mathrm{~mm}$ e $0,05 \mathrm{~mm})$, colocadas uma sobre a outra. Posteriormente, as amostras foram secas em estufa a uma temperatura entre $40^{\circ} \mathrm{C}$ e $50^{\circ} \mathrm{C}$ durante aproximadamente 48 horas e depois analisadas (MORATÓ, 2001). Durante todos os procedimentos realizados em laboratório, foram utilizados equipamentos de segurança, tais como luvas, máscaras e óculos de segurança, para evitar a contaminação por possíveis patógenos.

Todas as amostras foram analisadas a olho nu (MORATÓ, 2001). Os itens encontrados foram separados em diferentes categorias como penas, pelos, unhas, fragmentos de ossos, escamas, dentes e invertebrados (UCHOA; MOURA-BRITO, 2004; VIDOLIN, 2004). Para cada amostra examinada foi elaborada uma ficha que incluiu também a relação e contagem de itens alimentares identificados. A análise 
microscópica dos pelos foi realizada segundo a técnica descrita por Quadros e Monteiro-Filho (2006 a,b) e orientada pela chave identificação de espécies de pequenos mamíferos não voadores em agroecossistemas do Estado de São Paulo (MARTIN et al., 2009).

Para a identificação correta da amostra e de seu predador, assim como das presas do grupo dos mamíferos os pelos encontrados nas amostras fecais foram fotografados e analisados em microscopia eletrônica de varredura (Unesp/Botucatu) (Figura 2). Dentes, invertebrados, bicos e garras foram enviados a especialistas para refinar a análise e identificação.

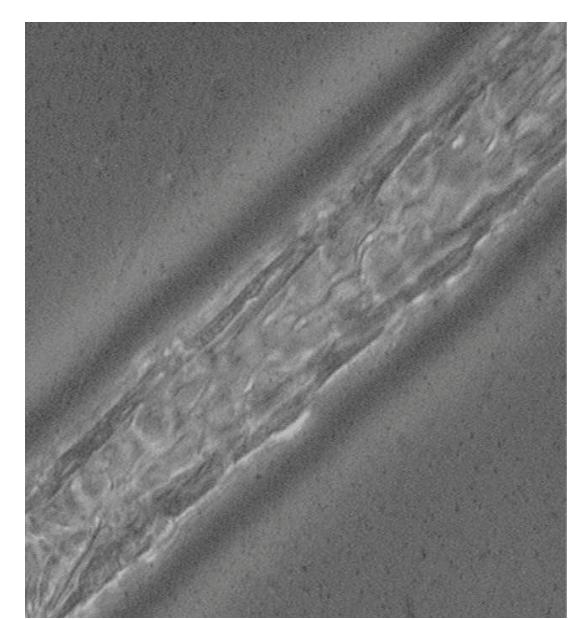

A

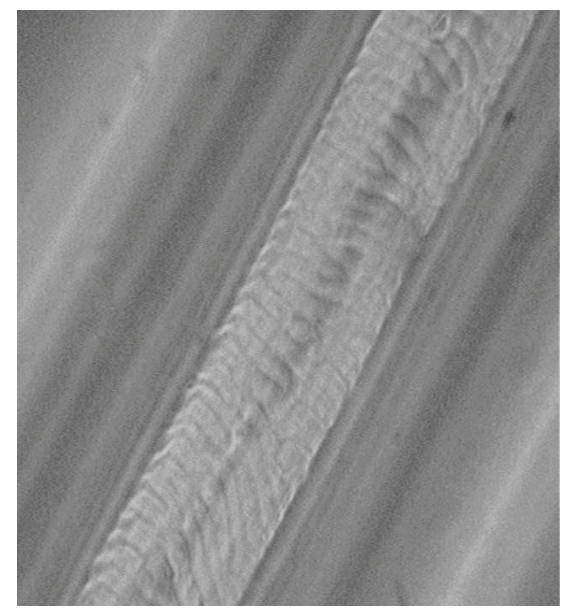

B

Figura 2. Microscopia eletrônica de varredura de cutícula de pelo guarda de Necromys lasiurus (a) e Didelphis albiventris (b) (x1000).

As fezes foram coletadas mensalmente com um esforço amostral aproximado de 24 horas mensais em campo, divididas em quatro dias de coleta, para garantir que toda a fazenda fosse revisada, e que a maior quantidade de amostras fosse coletada em cada campanha.

\subsection{METODOLOGIA ANALÍTICA}

Foram realizados cálculos relacionados à qualificação e quantificação da dieta de Puma concolor, tais como: 
a) quantidade de itens alimentares identificados (BISBAL, 1986), que será expressa pelo número de itens encontrados nas amostras fecais;

b) frequência de ocorrência (KREBS, 1989), que indica se o item é mais ou menos comum na dieta, e é calculada por meio da razão do número total de vezes que determinado item foi encontrado pelo número total de amostras coletadas;

c) biomassa consumida: foi utilizado como base o peso médio de cada item multiplicado pelo número mínimo comprovado de vezes em que aparece nas amostras de fezes (KREBS, 1999). Pela dificuldade de presumir a biomassa inteiramente consumida de espécies de mamíferos de médio porte, foi utilizado um valor médio do peso de um adulto (EISENBERG; REDFORD, 1992);

d) porcentagem de biomassa: que foi calculada pela divisão da massa do item estudado sobre a massa total de todos os itens consumidos (SANTOS, 1999);

e) amplitude do nicho alimentar: esta medida estima quantitativamente o grau de especialização da dieta de uma espécie, medindo a uniformidade de distribuição de indivíduos entre os recursos (KREBS, 1989, 1999).

A comparação da frequência absoluta dos itens consumidos por cada espécie, e sazonalmente dentro de cada espécie (estações seca (maio-setembro) e chuvosa (outubro-abril)) foram comparados por meio de teste qui-quadrado (SIMPSON et al., 1960).

Para que os dados não fossem superestimados alguns dos itens encontrados não foi utilizada nas análises estatística: invertebrados (este grupo não faz parte da dieta de $P$. concolor, podendo ser encontrado nas amostras por ingestão acidental ou contaminação da mesma) (MAGIOLI et al., 2014); sementes ( $P$. concolor não é considerado um dispersor de sementes, por isso não se realizaram testes germinativos e o item foi adicionado à categoria "material vegetal") (IRIARTE et al., 1990; MORENO et al., 2006; MAGIOLI et al., 2014); unhas (este item não foi analisado, pois se utilizou dos pelos e penas para a classificação de mamíferos e aves, pela baixa possibilidade de identificação que não seja por meio de análise de DNA, não entrando em nenhuma categoria). 
Os valores de frequência de ocorrência ofereceram informação sobre o impacto relativo que o predador provoca na comunidade de presas por ele consumidas, ao passo que com os dados de biomassa, pode-se ter uma avaliação mais acurada da importância relativa de cada item na dieta do próprio predador. Para verificar possíveis diferenças entre a frequência dos itens nas amostras foi utilizado teste $\mathrm{t}$ Student, e foi considerado significativo todo resultado com probabilidade igual ou inferior a $0,05(\mathrm{p}=<0,05)$. Os mamíferos com peso $<1 \mathrm{~kg}$ foram classificados como de pequeno porte; para médio porte utilizou-se $1-15 \mathrm{~kg}$ e $>15 \mathrm{~kg}$ para grande porte (IRIARTE et al., 1990). Para evitar a superestimação de presas foram consideradas certas estruturas para identificação e quantificação de cada táxon. Para pequenos roedores, maxilares contendo dentes foram contados, uma vez que esta estrutura proporciona a melhor estimativa da proporção da presa consumida (LOCKIE, 1959). Cada amostra coletada e analisada foi considerada como unidade amostral. Caso não houvesse a presença de crânios e/ou mandíbulas os itens foram qualificados por meio de análise tricológica. Os tatus foram identificados por meio da presença de pelos e placas dérmicas.

O peso médio de cada item foi obtido a partir de dados já coletados na área de estudo (MARTIN et al., 2012) e de coleções de referência ou, em alguns casos, a partir da literatura (EISENBERG; REDFORD, 1992). Não foi possível a identificação das penas encontradas nas fezes, portanto foi calculado um valor médio para a biomassa destas presas, quando as mesmas não foram identificadas pelos ornitólogos consultados. Por exemplo, para jacu (Penelope superciliaris) foi utilizado o peso médio de $1,5 \mathrm{~kg}$, uma vez que é a espécie de ave com maior frequência de ocorrência e probabilidade de ser predada pela onça parda na área de estudo (MILAN, 2013).

\section{RESULTADOS E DISCUSSÃO}

Foram analisadas 103 amostras de fezes de $P$. concolor, possuindo 12 categorias de itens de possível identificação, sendo: ossos, vegetal, penas, pelos, unhas/garras, sementes, pele, invertebrados, dentes, bicos, maxilar e outros. A categoria "outros" foi inclusa para abrigar itens não alimentares como material plástico (Tabela 1). Dentro da categoria vegetal o item "material vegetal", composto 
por uma miscelânea de folhas de espécies da família Poaceae (capim/pasto), cascas de frutas e outras folhas foram encontradas em $91,3 \%$ das amostras analisadas. A ingestão de folhas é comumente relatada por seu efeito positivo na digestão de muitos outros carnívoros, auxiliando também na eliminação de pelos das vias digestivas, mas também podem ser provenientes do conteúdo estomacal das presas (DIETZ, 1984; ARAGONA; SETZ, 2001; BUENO; MOTTA-JUNIOR, 2009).

Com relação aos itens alimentares de origem animal, não houve diferença significativa entre os itens alimentares $(t=4,33 \mathrm{p}=0,159)$, no entanto, as aves foram mais frequentes (74\%), seguidos pelos mamíferos (61\%), diferente do encontrado em outros estudos onde as presas mais frequentes são representantes do grupo dos mamíferos (ROHE, 2002; VIDOLIN, 2004). Millan (2013) descreve que paisagens silviculturais (plantio de eucalipto como matriz) podem atrair grande diversidade de aves silvestres, proporcionando-as abrigo e alimento, principalmente nos primeiros anos do plantio, o que pode explicar o grande consumo deste item na dieta de P. concolor. Dentro do grupo dos mamíferos os roedores Necromys lasiurus e Oligoryzomys spp. e o marsupial Didelphis albiventris foram as espécies mais frequentes na dieta de P. concolor (Figura 3).

Tabela 1. Quantidade e frequência de ocorrência em porcentagem dos itens encontrados na dieta de $P$. concolor

\begin{tabular}{lcc}
\hline \multicolumn{1}{c}{ Item } & $\begin{array}{c}\text { Número de fezes contendo } \\
\text { o item }\end{array}$ & Frequência de ocorrência (\%) \\
\hline Ossos & 103 & 100 \\
\hline Vegetal & 94 & 91.3 \\
\hline Penas & 76 & 74 \\
\hline Pelos & 63 & 61 \\
\hline Unhas & 63 & 61 \\
\hline Sementes & 36 & 35 \\
\hline Pele & 31 & 30 \\
\hline Invertebrados & 23 & 22 \\
\hline Outros & 18 & 17 \\
\hline Dentes & 14 & 13 \\
\hline Bicos & 14 & 13 \\
\hline Maxilar & 10 & 9,7 \\
\hline
\end{tabular}


As aves apresentaram a maior biomassa consumida $(114 \mathrm{~kg})$, diferindo de outros autores, onde a maior biomassa consumida pela espécie foi de mamíferos, seguida de répteis (MARTINS, 2008; VIDOLIN, 2004). Os mamíferos de médio porte apresentaram a segunda maior biomassa consumida (20 kg), sendo, principalmente, representados pela família Dasypodidae ( $42 \mathrm{~kg}$ ) (Dasypus novemcinctus e Cabassous tatouay). Os valores de porcentagem de biomassa apresentados na Figura 3 e Tabela 2 traduzem a proporção da biomassa consumida de cada item na dieta. As espécies de mamíferos de pequeno porte e de tatus consumidas pela espécie são comuns e abundantes na área de estudo (MARTIN et al., 2012). Quando comparados os valores de frequência de ocorrência das presas entre as estações do ano não foi encontrada diferença significativa $\left(x^{2}=2.878, \mathrm{p}=0,237\right)$.

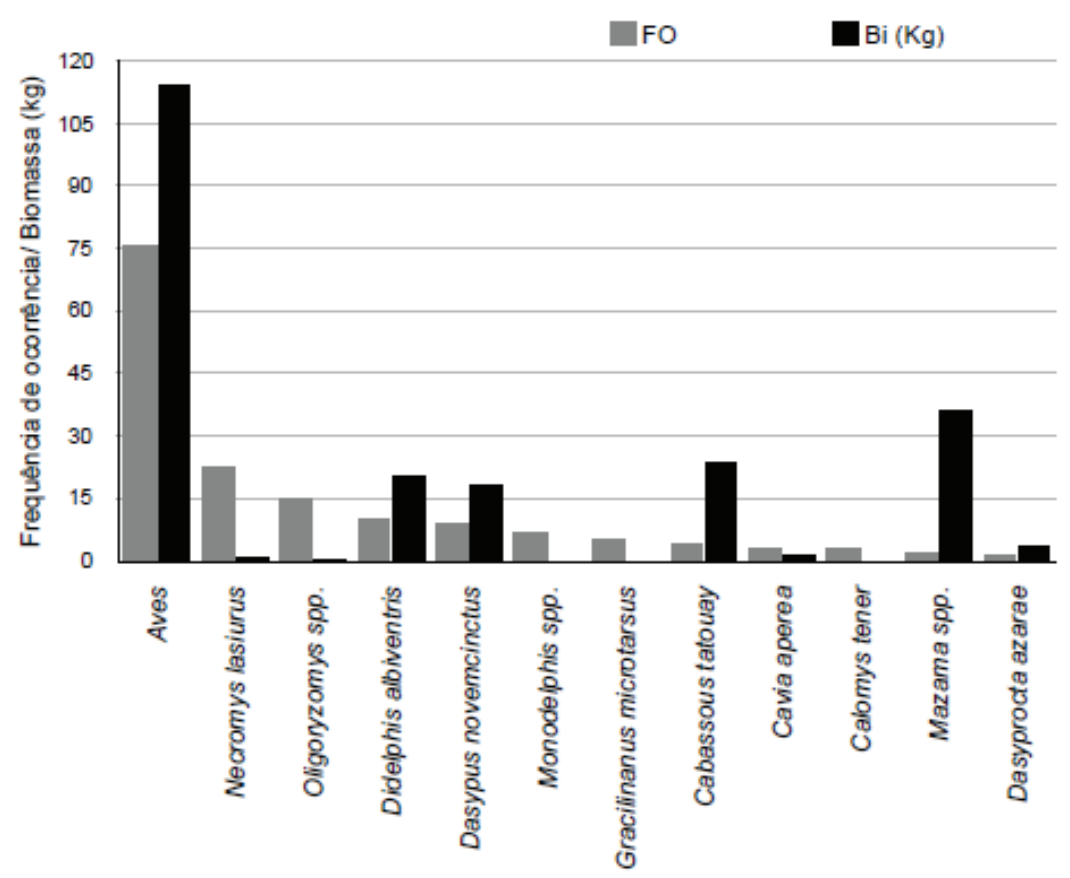

Figura 3. Frequência de ocorrência (FO) e biomassa consumida em $\mathrm{Kg}$ (Bi) dos itens alimentares encontrados nas amostras de fezes de Puma concolor 
Tabela 2. Frequência de ocorrência (FO) e Biomassa consumida em kg (Bi) de cada item identificado na dieta de onça parda (P. concolor) em uma paisagem agrícola

\begin{tabular}{lcc} 
Item & FO & Bi $\mathbf{K g})$ \\
Aves & 76 & 114 \\
\hline Necromys lasiurus & 22 & 1,03 \\
\hline Oligoryzomys spp. & 15 & 0,52 \\
\hline Didelphis albiventris & 10 & 20 \\
\hline Dasypus novemcinctus & 9 & 18 \\
\hline Monodelphis spp. & 7 & 0,28 \\
\hline Gracilinanus microtarsus & 5 & 0,17 \\
\hline Cabassous tatouay & 4 & 24 \\
\hline Cavia aperea & 3 & 1,8 \\
\hline Calomys tener & 3 & 0,11 \\
\hline Mazama spp. & 2 & 36 \\
\hline Dasyprocta azarae & 1 & 3,5
\end{tabular}

Estudos sobre a dieta de onça-parda em agroecossistemas ainda são escassos, se comparados a outros carnívoros como os canídeos, por exemplo, sendo a maioria dos trabalhos sobre dieta de $P$. concolor provenientes de áreas de unidades de conservação (VIDOLIN, 2004). Em agroecossistemas a onça-parda apresenta uma dieta especializada, com poucas presas predominantes, sendo os pequenos mamíferos o grupo mais frequente na dieta (NOVACK, 2003; MAGIOLI et al., 2014). Os dados deste estudo corroboram com o mencionado acima, uma vez que a onça parda usa de forma diferente os recursos alimentares existentes em determinadas épocas, ou seja, ela seleciona suas presas de acordo com a abundância destas no ambiente. No presente estudo, as aves foram o grupo mais consumido seguido dos pequenos mamíferos e dos tatus (Tabela 2).

O valor de amplitude de nicho para esta espécie no presente estudo foi de 0,37 , indicando uma baixa amplitude de nicho, ou seja, uma dieta especializada em vertebrados, corroborando com os trabalhos de Novack (2003) e Vidolin (2004). No entanto, esse valor pode variar de acordo com o ambiente estudado (0,24 - 
pantanal; 0,33 - Espírito Santo; 0,80 - Juréia, SP; 0,73 - cerrado) (OLIVEIRA, 2002; MARTINS et al., 2008), sendo assim a espécie pode apresentar padrões diferentes com relação à amplitude de nicho, uma vez que isso varia de acordo com a paisagem onde a espécie se encontra. Desta forma, a espécie mantém um padrão alimentar semelhante, alterando apenas a composição específica de cada comunidade de presas, e portanto sua estratégia de caça, corroborando com o estudo de Magiolli et al., (2014).

Segundo Emmons (1987) e Sunquist (1989), o consumo de presas mais abundantes pode estar relacionado a um comportamento oportunista de caça, e não necessariamente à especialização na captura das presas. Ou seja, as onças-pardas possuem características de uma espécie mais generalista quanto à preferência por presas, com uma grande plasticidade comportamental e ecológica que permite que elas consumam presas mais abundantes em cada situação. Segundo Magioli et al., (2014), por meio de análise isotópica da dieta de onça parda em agroecossistemas, constataram que a espécie consome majoritariamente presas provenientes da matriz agrícola. Podemos, desta forma, inferir que a onça-parda (P. concolor) tem capacidade de aclimatação ou até adaptação (abordagem genética) a ambientes agrosilvipastoril, podendo desta forma, alterarem sua dieta, sem prejudicar sua ecologia, dependendo do contexto e da paisagem (MIOTO et al., 2012).

A matriz da paisagem estudada sofreu grandes alterações ao longo dos anos, passando de uma matriz de pastagem para uma matriz de eucalipto, e esta se altera ao longo dos anos, passando de uma matriz aberta nos primeiros anos do plantio, para uma matriz fechada nos últimos anos do plantio. Estas alterações afetam diretamente os grupos de presas, principalmente as populações de pequenos mamíferos, alterando assim a composição específica dentro do plantio ao longo do ciclo do eucalipto. Nos primeiros anos as espécies dominantes são típicas de áreas abertas (Calomys tener, Necromys lasiurus), e com o passar do tempo são substituídas por espécies "florestais" como Oligoryzomys flavescens, Oligoryzomys nigripes, Gracilinanus microtarsus e Cryptonanus agricolai (MARTIN et al., 2012). As espécies de áreas abertas não desaparecem com o crescimento do eucalipto, apenas diminuem sua população uma vez que as áreas não apresentam as características desejadas. Desta forma, indiretamente, as alterações na paisagem 
provocam mudanças no uso do espaço pelas espécies de maior porte assim como em sua dieta.

A fragmentação de habitats naturais leva a espécie em estudo a migrar para áreas antropizadas como plantio de eucalipto e cana-de-açúcar, em busca de alimento e abrigo (VERDADE; CAMPOS, 2004; MAZOLLI, 2010; MIOTTO, 2011, 2012, MAGIOLI et al., 2014), uma vez que são os elementos mais comuns na matriz agrícola do Estado de São Paulo. O entendimento das alterações na ecologia das espécies é de grande importância na tomada de decisões relacionadas ao desenvolvimento econômico, social, cultural e ambiental de uma determinada região, assim como para medidas mitigadoras e de conservação. Sendo assim, dados como os do presente estudo são fundamentais para mostrar a importância das paisagens agrícolas na manutenção da espécie em questão, além de auxiliar na conservação de todas as espécies associadas a ela (DOTTA; VERDADE, 2011; GHELER-COSTA, et al., 2012, 2013; MARTIN et al., 2012). Informações sobre como a expansão das atividades humanas, como a agricultura, agem sobre espécies de topo de cadeia (espécies guarda-chuva) (ROBERGE; ANGELSTAM, 2004) podem contribuir para a melhora da compreensão e efetiva conservação global, uma vez que as ameaças antrópicas são ainda mais prejudiciais quando interagem com o risco intrínseco de extinção das espécies (CARDILLO et al., 2004, 2006; DOBROVOLSKI et al., 2013). Nesse caso, mesmo as melhores soluções de priorização podem não permitir conservação efetiva para carnívoros ou outros grupos taxonômicos sensíveis (VISCONTI et al., 2011).

\section{CONSIDERAÇÕES FINAIS}

A abordagem trófica fornece dados não só da ecologia do predador, mas também de toda a dinâmica das populações de presas, da relação predador-presa e ainda informações, mesmo que de forma indireta, sobre toda a cadeia trófica (DORRESTEIJN et al., 2015). Permitindo o monitoramento da diversidade de presas e predadores, além do entendimento do uso do espaço, biologia reprodutiva, carga parasitária e contato com patógenos (RAOUL et al., 2015; SRIVATHSAN et al., 2015; MARTINS et al., 2015). Estudos tróficos podem contribuir para o aumento da 
abordagem teórica sobre como a dinâmica dos ecossistemas são influenciados pelos predadores em paisagens com predomínio de atividades humanas (MARKOVCHICKNICHOLLS et al., 2008; RIPPLE et al., 2014).

A inserção do componente agrícola (paisagem agrícola) em projetos é urgente uma vez que esta paisagem abriga uma biodiversidade meritória de esforços conservacionistas, tais como a onça-parda e suas presas (DOTTA; VERDADE, 2011; GHELER-COSTA et al., 2012, 2013; VERDADE et al., 2015; MAGIOLI et al., 2016). Esta visão interdisciplinar dos ecossistemas pode contribuir para conciliar a produção alimentar e a conservação da biodiversidade, principalmente de mamíferos carnívoros, a fim de evitar os conflitos com produtores agrícolas.

\section{AGRADECIMENTOS}

Este estudo foi parcialmente financiado pelo projeto Fapesp (Proc. $\mathrm{N}^{\mathrm{O}}$ 2012/23897-3). Agradecemos ao Prof. Dr. Alexandre R. Percequillo (Esalq/USP), pelas identificações dos itens referentes a pequenos mamíferos encontrados na dieta da onça-parda. Ao Dr. Luis Miguel Rosalino (Universidade de Aveiro, Portugal), pelas inúmeras e importantes contribuições durante este estudo. E também a Suzano Papel e Celulose pela autorização de estudo nas áreas.

\section{REFERÊNCIAS}

ARAGONA, M.; SETZ, E. Z. Diet of the maned wolf, Chrysocyon brachyurus (Mammalia: Canidae), during wet and dry seasons at Ibitipoca State Park, Brazil. Journal of Zoology, v. 254, p. 131- 136, 2001.

BISBAL, F. J. Food habits of some neotropical carnivores in Venezuela (Mammalia, Carnívora). Mammalia, v. 50, n. 3, p. 329-339, 1986.

BUENO, A. A.; MOTTA-JUNIOR, J. C. Feeding habits of the maned wolf, Chysocyon brachyurus (Carnivora: Canidae), in southeast Brazil. Studies on Neotropical 
Fauna and Environment, v. 44, n. 2, p. 67-75, 2009.

CARDILLO, M.; MACE, G. M.; GITTLEMAN, J. L.; PURVIS, A. Latent extinction risk and future battlegrounds of mammal conservation. Proceedings of the National Academy of the United States of America, v. 103, p. 4157- 4161, 2006.

CARDILLO, M.; PURVIS, A.; SECHREST, W.; GITTLEMAN, J. L.; BIELBY, J.; MACE, G. M. Human population density and extinction risk in the world's carnivores. Plos Biology, v. 2, p. 909-914, 2004.

CRAWSHAW, P. G.; QUIGLEY, H. B. Food habitats of jaguars and cougars in the Pantanal, Brazil. In: MEDELLIN, R. A.; CHETKIEWICZ, C.; RABINOWITZ, A.; REDFORD, K. H.; ROBINSON, J. G.; SANDERSON, E.; TABER, A. Jaguars in the new millennium. National Autonomous University of Mexico, 2002. p., 223-235.

DIETZ, J. M. Ecology and social organization of the maned wolf (Chrysocyon brachyurus). Smithsonian Contributions to Zoology, Washington, v. 392, p. 1-51, 1984.

DOBROVOLSKI, R.; LOYOLA, R. D.; GUILHAUMON, F.; GOUVEIA, S. F.; DINIZ-FILHO, J. A. F. Global agricultural expansion and carnivore conservation biogeography. Biological Conservation, v. 165, p. 162-170, 2013.

DORRESTEIJN, I.; SCHULTNER, J.; NIMMO, D. G.; FISCHER, J.; HANSPACH, J.; KUEMMERLE, T.; RITCHIE, E. G. Incorporating anthropogenic effects into trophic ecology: predator-prey interactions in a human-dominated landscape. Proceedings of the Royal Society B, v. 282, p. 301-306, 2015.

DOTTA, G.; VERDADE, L. M. Medium to large-sized mammals in agricultural landscapes of South-eastern Brazil. Mammalia, v. 75, p. 345-352, 2011.

EISENBERG, J. F.; REDFORD, K. H. Mammals of the Neotropics. The Southern Cone: Chile, Argentina, Urugauy, Paraguay. University of Chicago Press, Illinois, USA, v. 2, 1992, 440p. 
EMMONS, L. H. Comparative feeding ecology of felids in a Neotropical Rainforest. Behavior Ecology and Sociobiolog y, v. 20, p. 271-283, 1987.

GHELER-COSTA, C.; SABINO-SANTOS J. R. G.; AMORIN, L. S.; ROSALINO, L. M.; FIGUEIREDO, L. T. M.; VERDADE, L. M. The effect of pre-harvest fire on the small mammal assemblage in sugarcane fields. Agriculture, Ecosystems and Environment, v. 171, p. 85-89, 2013.

GHELER-COSTA, C.; VETTORAZZI, C. A.; PARDINI, R.; VERDADE, L. M. The distribution and abundance of small mammals in agroecosystems of southeastern Brazil. Mammalia, v. 76, p. 185 - 191, 2012.

GONZALEZ-BORRAJO, N.; LOPEZ-BAO, J. V.; PALOMARES, F. Spatial ecology of jaguars, pumas, and ocelots: a review of the state of knowledge. Mammal review, v. 47, p. $62-75,2016$.

HEATH, M. R.; SPEIRS, D. C.; STEELE, J. H. Understanding patterns and processes in models of trophic cascades. Ecology letters, v. 17, p. 101-114, 2014.

IRIARTE, J. A.; FRANKLIN, W. L.; JOHNSON, W. E.; REDFORD, K. H. Biogeographic variation of food habits size of the America puma. Oecologia, v. 85, p.185-190, 1990.

KERTSON, B. N.; SPENCER, R. D.; MARZLUFF, J. M.; HEPINSTALL-CYMERMAN, J.; GRUE, C. Cougar space use and movements in the wildland-urban landscapeof western Washington. Ecological Applications, v. 21, p. 2866-2881, 2011.

KREBS, C. J. Ecological methodology. 2. ed. New York: Harper \& Row, 1999, 581p. KREBS, C. J. Ecological methodology. New York: Harper \& Row, 1989, 654p.

LOCKIE, J. D. The estimation of the food of foxes. Journal of Wildlife Management, v. 23, p, 224-227, 1959.

MAGIOLI, M.; FERRAS, K. M. P. M. B; SETZ, E. Z. F.; PERCEQUILLO, A. R.; RONDON, M. V. D. S. S.; KUHNEN, V. V.; DO PRADO, H. A. Connectivity maintain mammal 
assemblages functional diversity within agricultural and fragmented landscapes. European Journal of Wildlife Research, v. 62, p.1-16, 2016.

MAGIOLI, M.; MOREIRA, M. Z.; FERRAZ, K. M. B.; MIOTTO, R. A.; CAMARGO, P. B.; RODRIGUES, M. G.; CANHOTO, M. C. S.; SETZ, E. F. Stable Isotope Evidence of Puma concolor (Felidae) Feeding Patterns in Agricultural Landscapes in Southeastern Brazil. Biotropica, v. 46, n. 4, p.451-460, 2014.

MARKOVCHICK NICHOLLS, L.; REGAN, H. M.; DEUTSCHMAN, D. H.; WIDYANATA, A.; MARTIN, B.; NOREKE, L.; HUNT, T. A. Relationships between human disturbance and wildlife land use in urban habitat fragments. Conservation Biology, v. 22, p. 99-109, 2008.

MARTIN, P. S.; GHELER-COSTA, C.; LOPES, P. C.; ROSALINO, L. M.; VERDADE, L. M. Terrestrial non-volant small mammals in agro-silvicultural landscapes of Southeastern Brazil. Forest, Ecolog y and Management, v. 282, p. 185-195, 2012.

MARTIN, P. S.; GHELER-COSTA, C.; VERDADE, L. M. Microestruturas de pelos de pequenos mamíferos não-voadores: chave para identificação de espécies de agroecossistemas do Estado de São Paulo, Brasil. Biota Neotropica, v. 9, 2009.

MARTINELLI, L.; FILOSO, S. Expansion of sugarcane ethanol production in Brazil: environmental and social challenges. Ecological Applications, v. 18, p. 885-898, 2008.

MARTINS, R.; QUADROS, J.; MAZZOLI, M. Hábito Alimentar e interferência antrópica na atividade de marcação territorial do Puma concolor e Leopardus pardalis (Carnivora: Felidae) e outros carnívoros na Estação Ecológica de Juréia-Itatins, São Paulo. Brasil. Revista Brasileira de Zoologia, v. 25, n. 3, p. 427-435, 2008.

MARTINS, T. F.; DINIZ-REIS, T. R.; LIBARDI, G. S.; PERCEQUILLO, A. R.; VERDADE, L. M.; MATUSHIMA, E. R.; LABRUNA, M. B. Ticks (Acari: Ixodidae) identified from preypredator interactions via faecal analysis of Brazilian wild carnivores. Experimental and Applied Acarology, v. 66, p. 119-125, 2015. 
MAZZOLLI, M. Mosaics of exotic forest plantations and native forests as habitat of pumas. Environmental Management, v. 46, n. 2, p. 237-253, 2010.

MILLAN, C. H. Influência de práticas de manejo e contexto da paisagem sobre a ocorrência de aves em plantio exótico de eucalipto. 2013. 43f. Dissertação de mestrado (Mestrado em Ecologia) - Universidade de São Paulo, São Paulo, 2013.

MIOTTO, R. A.; CERVINI, M.; BEGOTTI, R. A.; GALETTI JR, P. M. Genetic diversity and population structure of pumas (Puma concolor) in southeastern Brazil: implications for conservation in a human-dominated landscape. Conservation Genetics, v. 12, n. 6, p. 1447-1455, 2011.

MIOTTO, R. A.; CERVINI, M.; BEGOTTI, R. A; GALETTI JR, P. M. Monitoring a Cougar (Puma concolor) Population in a Fragmented Landscape in Southeast Brazil. Biotropica, v. 44, (1) p. 98-104, 2012.

MIOTTO, R. A.; RODRIGUES, F. P.; CIOCHETI, G.; GALETTI, P. M. Determination of the minimum population size of pumas (Puma concolor) through fecal DNA analysis in two protected Cerrado areas in the Brazilian southeast. Biotropica, v. 39, p. 647 654. 2007.

MORATÓ, D. Q. Seletividade e sazonalidade das presas consumidas pelo loboguará (Chrysocyon brachyurus) no parque nacional da Serra da Canastra, Minas Gerais. 2001. 86f. Dissertação (Mestrado em Ecologia) - Instituto de Biociências, Universidade de São Paulo, São Paulo, 2001.

MORENO, R. S.; KAYS, R. W.; SAMUDIO, R. Competitive release in diets of ocelot (Leopardus pardalis) and puma (Puma concolor) after jaguar (Panthera onca) decline. Journal of Mammalogy, v. 87, p. 808-816, 2006.

NOVACK, A. J. Impacts of subsistence hunting on the foraging ecology of jaguar and puma in the Maya Biosphere Reserve, Guatemala. 2003. 130f. Tese (Doutorado). Universidade da Flórida, Gainsville, 2003.

OLIVEIRA, T. G. Ecología comparativa de la alimentación del jaguar y del puma en el 
neotrópico. In: MEDELLIN, R. A.; CHETKIEWICZ, C.; RABINOWITZ, A.; REDFORD, K. H.; ROBINSON, J. G.; SANDERSON, E.; TABER, A. El Jaguar en el nuevo milenio. Una evaluacion de su estado, deteccion de prio- ridades y recomendaciones para la conservacion de los jaguares en America. México: Universidad Nacional Autonoma de Mexico, 2002, p.265-288.

QUADROS, J.; MONTEIRO-FILHO, E. L. A. Coleta e preparação de pelos de mamíferos para identificação em microscopia óptica. Revista Brasileira de Zoologia, v. 23, n. 1, p. 274-278, 2006 (a).

QUADROS,J.;MONTEIRO-FILHO,E.L.A. Revisãoconceitual, padrões microestruturais e proposta nomenclatória para os pelos-guarda de mamíferos brasileiros. Revista Brasileira de Zoologia, v. 23, n. 1, p. 279-296, 2006 (b).

RABINOWITZ, A. R.; WALKER, S. R. The carnivore community in a dry tropical forest mosaic in a Huai Hha Hhaeng Wildlife Sanctuary, Thailand. Journal of Tropical Ecology, v. 7, n. 1, p. 37-47, 1991.

RAOUL, F.; HEGGLIN, D.; GIRAUDOUX, P. Trophic ecology, behaviour and host population dynamics in Echinococcus multilocularis transmission. Veterinary parasitology, v. 213, p. 162-171, 2015.

RIPPLE, W. J.; ESTES, J. A.; BESCHTA, R. L.; WILMERS, C. C.; RITCHIE, E. G.; HEBBLEWHITE, M.; SCHMITZ, O. J. Status and ecological effects of the world's largest carnivores. Science, v. 343, p. 1241484, 2014.

ROBERGE, J. M.; ANGELSTAM, P. E. R. Usefulness of the umbrella species concept as a conservation tool. Conservation Biology, v. 18, p. 76-85, 2004.

ROHE, F. Hábitos alimentares da suçuarana (Puma concolor Linnaeus 1771) em mosaico de Floresta Secundária e reflorestamento de Eucaliptus saligna, em Mata Atlântica, no Município de Pilar do Sul/SP. 2002. 76f. Trabalho de Conclusão do Curso (Monografia) - Instituto de Biociências, Universidade Estadual Paulista, Rio Claro, 2002. 
RONDININI, C. et al. Global habitat suitability models of terrestrial mammals. Philosophical Transactions of the Royal Society B, v. 366, p. 2633-2641, 2011.

SANDOM, C. J.; DALBY, L.; FLØJGAARD, C.; KISSLING, W. D.; LENOIR, J.; SANDEL, B.; SVENNING, J.C. Mammal predator and prey species richness are strongly linked at macroscales. Ecology, v. 94, p.1112-1122, 2013.

SANTOS, E. F. Ecologia alimentar e dispersão de sementes pelo lobo-guará (Chrysocyon brachyurus, Illiger, 1811) em uma Área Rural no Sudeste do Brasil (Carnivora: Canidae). 1999. 68 f. Dissertação (Mestrado em Ciências Biológicas) Instituto de Biociências, Universidade Estadual Paulista, Rio Claro, 1999.

SANTOS, M. F. M.; PELLANDA, M.; TOMAZZONI, A. C.; HASENACK, H.; HARTZ, S. M. Mamíferos carnívoros e sua relação com a diversidade de hábitats no Parque Nacional dos Aparados da Serra, sul do Brasil. Iheringia, Série Zoologia, Porto Alegre, v. 94, p. 235-245, 2004.

SCHIPPER, J. et al. The status of the world's land and marine mammals: diversity, threat, and knowledge. Science v. 322, p. 225-230, 2008.

SCOGNAMILLO, D.; MAXIT, I. E.; SUNQUIST, M.; POLISAR, J. Coexistence of jaguar (Panthera onca) and puma (Puma concolor) in a mosaic landscape in the Venezuelan llanos. Journal of Zoology, v. 259, p. 269-279, 2003.

SIMPSON, G. G.; ROE, A.; LEWONTIN, R. Quantitative Zoology. Harcourt Brace and Company, New York, Burlingame, v. 1, 1960, 440p.

SRIVATHSAN, A.; SHA, J.; VOGLER, A. P.; MEIER, R. Comparing the effectiveness of metagenomics and metabarcoding for diet analysis of a leaf-feeding monkey (Pygathrix nemaeus). Molecular ecology resources, v. 15, p. 250-261, 2015.

SUNQUIST, M. E.; SUNQUIST, F. C. SUNQUIST. Ecological constrains on predation by large felids. In: GITTLEMAN, J. L. Carnivore behavior, ecology, and evolution. Ithaca: Cornell University Press, 1989, p. 283-301.

TERBORGH, J.; ESTES, J. A.; PAQUET, P.; RALLS, K.; BOYD-HEGER, D.; MILLER, B. 
J.; NOSS, R. F. The Role of Top Carnivores in Regulating Terrestrial Ecosistemys. In: TERBORGH, J.; SOULÈ, M. E. Continental conservation: Scientific foundations for regional conservation networks. Washington: Island Press, 1999, p. 39-64.

UCHOA, T.; MOURA-BRITTO, M. Hábito Alimentar e Uso do Habitat por Canídeos no Parque Estadual do Cerrado: avaliação da situação atual da família Canidae no limite sul do bioma Cerrado no Brasil. Cadernos da Biodiversidade, v. 4, n. 2, p. 59-65, 2004.

VERDADE, L. M.; CAMPOS, C. B. How much is a puma worth? Economic compensation as an alternative for the conflict between wildlife conservation and livestock production in Brazil. Biota Neotropica, v. 4, n. 2, p.1-4, 2004.

VERDADE, L. M.; GHELER-COSTA, C.; PENTEADO, M.; DOTTA, G. The Impacts of Sugarcane Expansion on Wildlife in the State of São Paulo, Brazil. Journal of Sustainable Bioenergy Systems, v. 2, p. 138-144, 2012.

VERDADE, L.M.; PENTEADO, M.; GHELER-COSTA, C.; DOTTA, G.; ROSALINO, L.M.; PIVELLO, V.R.; LYRA-JORGE, M.C. The conservation value of agricultural landscapes. In: VERDADE, L.M.; LYRA-JORGE, M.C.; PIÑA, C.I. Applied ecology and human dimensions on biological conservation. Springer-Verlag, Heidelberg: Germany, 2014, p.91-102.

VIDOLIN, G. P. Aspectos Bio-Ecológicos de Puma concolor (Linnaeus, 1771), Leopardus pardalis (Linnaeus, 1758) e Leopardus tigrinus (Schreber, 1775) na reserva natural Salto Morato, Guaraqueçaba, Paraná, Brasil. 2004. 89f. Dissertação (Mestrado em Conservação da natureza) - Universidade Federal do Paraná, Curitiba, 2004.

VISCONTI, P.; PRESSEY, R. L.; GIORGINI, D.; MAIORANO, L.; BAKKENES, M.; BOITANI, L.; ALKEMADE, R.; FALCUCCI, A.; CHIOZZA, F.; RONDININI, C. Future hotspots of terrestrial mammal loss. Philosophical Transactions of the Royal Society B, v. 366, p. 2693-2702, 2011.

WILLIAMS, R. J.; MARTINEZ, N. D. Limits to trophic levels and omnivory in complex food webs: theory and data. The American Naturalist, v. 163, p. 458-468, 2004. 
WOLFF, F. Vertebrate ecology in caatinga: Distribuition of wildlife relation to water. B. Diet of pumas (Puma concolor) and relative abundance of felids. 2001. 74 f. Tese (Mestrado) - University of Missouri-St. Louis, St. Louis, 2001.

Recebido em: 2016-02-09 Aceito em: 2017-02-10 